\title{
Abstract integration, Combinatorics of Trees and Differential Equations
}

\author{
Massimiliano Gubinelli \\ CEREMADE, Université Paris Dauphine, France \\ gubinelli@ceremade.dauphine.fr
}

September 2008

\begin{abstract}
This is a review paper on recent work about the connections between rough path theory, the Connes-Kreimer Hopf algebra on rooted trees and the analysis of finite and infinite dimensional differential equation. We try to explain and motivate the theory of rough paths introduced by $\mathrm{T}$. Lyons in the context of differential equations in presence of irregular noises. We show how it is used in an abstract algebraic approach to the definition of integrals over paths which involves a cochain complex of finite increments. In the context of such abstract integration theories we outline a connection with the combinatorics of rooted trees. As interesting examples where these ideas apply we present two infinite dimensional dynamical systems: the Navier-Stokes equation and the Korteweg-de-Vries equation.
\end{abstract}

Keywords: Rough Path theory, Connes-Kreimer Hopf algebra, driven differential equations, Navier-Stokes equation, Korteweg-de-Vries equation.

\section{Introduction}

Rough path theory has been developed by T. Lyons for the analysis of the map $\Phi: x \mapsto y$ which sends a vector-valued driving signal $x$ to the solution of the differential equation

$$
d y_{t}=\sum_{a} f_{a}\left(y_{t}\right) d x_{t}^{a}, \quad y_{0}=\bar{y}
$$

where $\bar{y}$ is the inital condition. This equation has a well defined meaning as a non-autonomous ODE when $x_{t}$ is differentiable in $t$. In applications however it is interesting to consider more general driving signals, e.g. when taking into account random perturbations of dynamical systems where $x_{t}$ is the Brownian motion which is almost surely non-differentiable. Indeed the application to stochastic differential equations (SDEs) has been the main motivation in the developments of the theory. The standard approach is then to understand eq. (11) as the integral equation

$$
y_{t}=\bar{y}+\int_{0}^{t} \sum_{a} f_{a}\left(y_{s}\right) d x_{s}^{a}
$$


provide a well-defined meaning for the stochastic integral in the r.h.s. and the proceed to solve the equation by standard fixed-point methods. Usually the integral above can be an Itô integral or a Stratonovich one, but other more exotic choices are possible in other stochastic contexts (e.g. the Skorohod integral, the "normal ordered" integral, etc...). Whenever we speak of a solution $y$ to the SDE (11) we mean suitable random function $y$ for which $f(y)$ can be integrated against $d x$ in an appropriate sense.

Lyons' basic observation was that, in the case of a smooth control $x$, the solution of the differential equation is a well-behaved function of the iterated integrals $X$ of $x$ :

$$
X_{t s}^{\bar{a}}=\int_{s}^{t} \int_{s}^{u_{n}} \cdots \int_{s}^{u_{2}} d x_{u_{1}}^{a_{1}} \cdots d x_{u_{n}}^{a_{n}}
$$

where we denote with $\bar{a}$ the multi-index $\left(a_{1}, \ldots, a_{n}\right)$ with $|\bar{a}|=n$ its length. In some sense the iterated integrals encode the local behaviour of the path $x$ well enough to faithfully recover its effect on the solution $y$. In a system perspective, the iterated itegrals provide a canonical set of coordinates for the analysis of non-linear systems much like the standard Fourier coefficients are natural coordinates for linear ones.

Like Fourier coefficients, iterated integrals enjoy nice relations upon concatenation of paths: given three times $s<u<t$ we have the celebrated Chen relations [1] between iterated integrals:

$$
X_{t s}^{a_{1} \cdots a_{n}}=X_{t u}^{a_{1} \cdots a_{n}}+X_{u s}^{a_{1} \cdots a_{n}}+\sum_{k=1}^{n-1} X_{t u}^{a_{1} \cdots a_{k}} X_{u s}^{a_{k+1} \cdots a_{n}} .
$$

These non-linear equations plays a fundamental role in the development of the theory.

The plan of the paper is the following: in Sect. 2 we motivate the simplest instance of a rough path in the context of integration against smooth approximations of an irregular path. Sect. 3 we describe an algebraic approach to integration and its use to define integrals against a rough path. This approach is not standard from the point of view of rough paths. More conventional expositions of the theory can be found in 2, 3] and [4. In Sect. 4 we describe the combinatorics of iterated integrals of very general type via the Connes-Kreimer Hopf algebra on rooted trees following $[5$. This will ultimately allow to define and solve differential equation associated to such integrals. Finally in Sect. 5 we apply the objects and the related combinatorics of algebraic integrals to the study of infinite dimensional differential equations via two prototypical examples: the $3 \mathrm{~d}$ Navier-Stokes equation as studied in [6] and the 1d periodic Korteweg-de-Vries equation [7].

\section{Renormalizable theories of integration}

The analysis of the integral equation (2) in the case when $x$ is a non-differentiable function can be split in two parts: the definition of the integral on the r.h.s. and the fix-point argument. Let us concentrate on the first part. Assume given a continuous function $x:[0, T] \rightarrow \mathbb{R}^{n}$ which we assume only $\gamma$-Hölder continuous, i.e. for which the following estimate holds

$$
\left|x_{t}-x_{s}\right| \leq C|t-s|^{\gamma}, \quad t, s \in[0, T]
$$

(where the least constant $C$ define the Hölder norm $\|x\|_{\gamma}$ ) and for simplicity let us restrict in this section to the case $1 / 3<\gamma<1$. Let us pose the problem of giving a "reasonable" definition of the 
integral

$$
I[\varphi]_{t s}=\int_{s}^{t} \varphi\left(x_{u}\right) d x_{u}
$$

for some smooth one-form $\varphi: \mathbb{R}^{n} \rightarrow \mathbb{R}^{n}$. Proceeding by approximation we consider a family $\{x(\varepsilon)\}_{\varepsilon>0}$ of smooth approximations to the path $x$ and let

$$
I[\varphi](\varepsilon)_{t s}=\int_{s}^{t} \varphi\left(x(\varepsilon)_{u}\right) d x(\varepsilon)_{u} .
$$

It is easy to convince ourselves that in general we do not have any control of these integrals if $x(\varepsilon) \rightarrow x$ when $\varepsilon \rightarrow 0$ in the $\gamma$-Hölder norm. It is then a remarkable fact that all the possible integrals obtained varying the function $\varphi$ all converge at the same time (provided $\varphi$ is sufficiently smooth) when the approximated iterated integral of order two:

$$
X(\varepsilon)_{t s}^{a_{1} a_{2}}=\int_{s}^{t} \int_{s}^{u} d x(\varepsilon)_{v}^{a_{1}} d x(\varepsilon)_{u}^{a_{2}}
$$

converges to a function $X:[0, T]^{2} \rightarrow \mathbb{R}^{n} \otimes \mathbb{R}^{n}$ in the sense that

$$
\sup _{0 \leq s<t \leq T} \frac{\left|X(\varepsilon)_{t s}^{a_{1} a_{2}}-X_{t s}^{a_{1} a_{2}}\right|}{|t-s|^{2 \gamma}} \rightarrow 0 \quad \text { as } \varepsilon \rightarrow 0 .
$$

So in some sense we can claim that the integration theory agains the path $x$ is well defined as long as we are able to control the convergence of the iterated integral of order two $X^{a_{1} a_{2}}$ : all the other integrals (and more fundamentally also all the higher order iterated integrals) will turn out to be nice functions of the data given by the path $x$ and $X^{a_{1} a_{2}}$.

It is suggestive to understand this phenomenon as a very simple example of "renormalizable theory" where all the quantities of interests have well defined meanings as functionals of a finite number of fundamental objects whose intrinsic determination remains outside the scope of the theory itself.

In this limited context we face the appeareance of the simplest non-trivial example of a rough path: the couple $\left(X^{a}, X^{a_{1} a_{2}}\right)$ where $X_{t s}^{a}=x_{t}^{a}-x_{s}^{a}$ is a $\gamma$-rough path, i.e. a path and some additional information in the form of "iterated integrals", for which a complete theory of integration and differential equations can be constructed (as we will see shortly). The fact that it is enough to consider only the second order integral is due to our hypothesis that $\gamma>1 / 3$.

Note that once the limit has been taken the object $X^{a_{1} a_{2}}$ is no more an iterated integral (in a classical sense) and can be characterized more abstractly by the following two properties

1. the Chen relation:

$$
X_{t s}^{a_{1} a_{2}}=X_{t u}^{a_{1} a_{2}}+X_{u s}^{a_{1} a_{2}}+X_{t u}^{a_{1}} X_{u s}^{a_{2}}
$$

2. a regularity condition

$$
\sup _{0 \leq s<t \leq T} \frac{\left|X_{t s}^{a_{1} a_{2}}\right|}{|t-s|^{2 \gamma}}<\infty .
$$

and more interestingly there could be more than one possible choice for this object compatible with these two conditions leading to different integration theories. 


\section{Algebraic integration}

To understand how the iterated integrals $X$ comes into play in the definition of $I[\varphi]$ we need some tool which allows us to analyze the "local" (with respect to the parameter) behaviour of the integral. Specifically we want to expand the integral in a short interval as

$$
I[\varphi]_{t s}=\varphi_{a}\left(x_{s}\right) X_{t s}^{a}+\partial_{b} \varphi_{a}\left(x_{s}\right) X_{t s}^{b a}+r_{t s}
$$

where $r$ stands for some remainder term which we hope will be of higher order in $|t-s|$ than the other terms. The main property of the integral $I[\varphi]$ is its trivial behaviour under splitting of the integration interval: $I[\varphi]_{t s}=I[\varphi]_{t u}+I[\varphi]_{u s}$ for $s<u<t$, this of course means also that the integral can be written as the increment of a function $I[\varphi]_{t s}=f_{t}-f_{s}$ (for example taking $f_{t}=I[\varphi]_{0 t}$ ). In $[8]$ we introduced a cochain complex $\left(\mathcal{C}_{*}, \delta\right)$ which encodes this basic property of integrals. For $n \geq 1$ let $\mathcal{C}_{n}$ be set of functions $a \in C\left([0, T]^{n} ; \mathbb{R}\right)$ such that $a_{t_{1} \cdots t_{n}}=0$ if $t_{i}=t_{i+1}$ for some $1 \leq i \leq n-1$. Elements in $\mathcal{C}_{n}$ will be called $n$-increments. Define a coboundary $\delta: \mathcal{C}_{n} \rightarrow \mathcal{C}_{n+1}$ as $\delta a_{t s}=a_{t}-a_{s}$ for $a \in \mathcal{C}_{1}, \delta a_{\text {tus }}=a_{t s}-a_{t u}-a_{u s}$ for $a \in \mathcal{C}_{2}$ and so on. Let $\mathcal{Z C}_{n}=\operatorname{Ker} \delta \cap \mathcal{C}_{n}$ and $\mathcal{B C}_{n}=\operatorname{Im} \delta \cap \mathcal{C}_{n}$. It is easy then to verify that $\delta \delta=0$ and that the complex $\left(\mathcal{C}_{*}, \delta\right)$ is exact, i.e. that $\operatorname{Im} \delta=\operatorname{Ker} \delta$ at any $\mathcal{C}_{n}, n \geq 1$. In particular a 2 -increment $a \in \mathcal{C}_{2}$ is the increment of a function $f \in \mathcal{C}_{1}$ if and only if $\delta a=0$. So at $\mathcal{C}_{2}$ the coboundary measures the degree of "exactness" of 2-increments. Moreover a key fact is the following: if $\delta a$ is suitably small then there exists only one function $f$ (modulo constants) such that

$$
\delta f=a+r
$$

where the remainder is small. To be more precise we need to introduce the relevant notion of "smallness". We say that $a \in \mathcal{C}_{2}^{\gamma}$ if

$$
\|a\|_{\gamma}:=\sup _{s<t} \frac{\left|a_{t s}\right|}{|t-s|^{\gamma}}<\infty
$$

And similarly $b \in \mathcal{C}_{3}^{\gamma}$ if

$$
\|b\|_{\gamma}:=\sup _{s<u<t} \frac{\left|b_{t u s}\right|}{|t-s|^{\gamma}}<\infty .
$$

Both $\mathcal{C}_{2}^{\gamma}$ and $\mathcal{C}_{3}^{\gamma}$ are Banach spaces when endowed with the norms $\|\cdot\|_{\gamma}$. Define $\mathcal{C}_{n}^{1+}=\cup_{z>1} \mathcal{C}_{n}^{z}$. Moreover if $g \in \mathcal{C}_{n}$ and $h \in \mathcal{C}_{m}$ then we write $g h$ for the element of $\mathcal{C}_{n+m-1}$ such that $(g h)_{t_{1} \cdots t_{n+m-1}}=$ $g_{t_{1} \cdots t_{n}} h_{t_{n} \cdots t_{n+m-1}}$.

The key result in this theory of increments is the existence of a sewing map which provide a natural inverse operation to $\delta$ :

Theorem 3.1 (The sewing map). There exists a unique bounded and linear map $\Lambda: \mathcal{Z C}_{3}^{1+} \rightarrow \mathcal{C}_{2}^{1+}$ such that $\delta \Lambda h=h$ for any $h \in \mathcal{Z C}_{3}^{1+}$.

In particular the map $(1-\Lambda \delta): \mathcal{C}_{2} \rightarrow \mathcal{C}_{2}$ projects (in essentially a unique way) suitable elements of $\mathcal{C}_{2}$ to $\mathcal{Z C}_{2}=\mathcal{B C}_{1}$ : the space of increments of functions. Then going back to the decomposition (8) and assuming that $\delta a \in \mathcal{C}_{3}^{1+}$ we can form the exact 2-increment $a-\Lambda \delta a=\delta f$ and obtain that the remainder $r$ is given by $\Lambda \delta a \in \mathcal{C}_{2}^{1+}$. It is also easy to see that, the decomposition (8) is unique if we require $r \in \mathcal{C}_{2}^{1+}$, indeed if two such decomposition exists, $(f, r)$ and $\left(f^{\prime}, r^{\prime}\right)$, their difference satisfy $\delta\left(f-f^{\prime}\right)=r-r^{\prime} \in \mathcal{C}_{2}^{z}$ for some $z>1$ and $f-f^{\prime}$ would be a function of $z$-Hölder class ans since $z>1$ that this function would take the constant value zero. 
With this notions at hand we realize that eq. (77) is nothing more than an instance of a decomposition similar to (8). As we have already seen we can determine both $I[\varphi]$ and $r$ at once using only the well-defined remaining terms in the r.h.s., so letting $a_{t s}=\varphi_{a}\left(x_{s}\right) X_{t s}^{a}+\partial_{b} \varphi_{a}\left(x_{s}\right) X_{t s}^{b a}$, in order to be able to apply $\Lambda$, we need to require that $\delta a \in \mathcal{C}_{3}^{1+}$. By some easy computation using the fact that $\delta$ satisfy some kind of Leibniz rule, we get

$$
\delta a_{t u s}=-\delta \varphi_{a}(x)_{t u} X_{u s}^{a}-\delta \partial_{b} \varphi_{a}(x)_{t u} X_{t s}^{b a}+\partial_{b} \varphi_{a}\left(x_{s}\right) \delta X_{t u s}^{b a}
$$

If we now exploit the Chen relation for $X^{b a}$ we can simplify this expression further

$$
\delta a_{t u s}=-\left[\delta \varphi_{a}(x)_{t u}-\partial_{b} \varphi_{a}\left(x_{s}\right) \delta X_{t u}^{b}\right] X_{u s}^{a}-\delta \partial_{b} \varphi_{a}(x)_{t u} X_{t s}^{b a}
$$

Assuming that $\varphi$ is sufficiently smooth $\left(C^{2}\right.$ is enough) we obtain easily that

$$
\delta \varphi_{a}(x)-\partial_{b} \varphi_{a}(x) \delta X^{b} \in \mathcal{C}_{2}^{2 \gamma}, \quad \delta \partial_{b} \varphi_{a}(x) \in \mathcal{C}_{2}^{\gamma}
$$

so that taking into account all the regularities we end up with $\delta a \in \mathcal{C}_{3}^{3 \gamma}$. Then our assumption on $\gamma$ ensure that $3 \gamma>1$ and we can prove that there exists a unique couple $(f, r)$ with $f \in \mathcal{C}_{1}$ and $r \in \mathcal{C}_{2}^{1+}$ such that

$$
\delta f=\varphi_{a}\left(x_{s}\right) X_{t s}^{a}+\partial_{b} \varphi_{a}\left(x_{s}\right) X_{t s}^{b a}+r_{t s} .
$$

By construction $f$ depends only on the $\gamma$-rough path $X$ and on $\varphi$. We can then define the integral by

$$
I[\varphi]=\delta f=(1-\Lambda \delta)\left[X^{a} \varphi_{a}(x)+X^{b a} \partial_{b} \varphi_{a}(x)\right] .
$$

To motivate the fact that the decomposition (10) is a sort of renormalized integral we can make the following observation. Take a partition $\left\{t_{i}\right\}$ of $[s, t]$ of size $\Delta$ and consider the Riemman sums $S_{\Delta}=\sum_{i} \varphi\left(x_{t_{i}}\right) X_{t_{i+1} t_{i}}$. In general we have no mean to say that these sums are convergent as $\Delta \rightarrow 0$. However given a $\gamma$-rough path $X$ we can perform a subtraction to these sums and define

$$
S_{\Delta}^{\prime}=\sum_{i}\left[\varphi_{a}\left(x_{t_{i}}\right) X_{t_{i+1} t_{i}}^{a}+\partial_{b} \varphi_{a}\left(x_{t_{i}}\right) X_{t_{i+1} t_{i}}^{b a}\right]
$$

then using the decomposition 10 as $\Delta \rightarrow 0$ we have the limit

$$
S_{\Delta}^{\prime}=\sum_{i}(\delta f)_{t_{i+1} t_{i}}-\sum_{i} r_{t_{i+1} t_{i}}=(\delta f)_{t s}-\sum_{i} o\left(\left|t_{i+1} t_{i}\right|\right) \rightarrow(\delta f)_{t s}
$$

since the first sum telescopes and the second is easily show to converge to zero.

Moreover the regular dependence on the data ensure that smooth approximations $I[\varphi](\varepsilon)$ converge to $I[\varphi]$ as here defined as long as we can prove the convergence of approximating path (and its second order iterated integrals) to the rough path $X$.

Exploiting the sewing map, the integral equation (2) in presence of a $\gamma$-rough path with $\gamma>1 / 3$ can be understood as a fixed-point equation for an unknown $y \in \mathcal{C}_{1}$ :

$$
\delta y=(1-\Lambda \delta)\left[X^{a} \varphi_{a}(y)+X^{a c} \partial_{b} \varphi_{a}(y) \varphi_{c}^{b}(y)\right]
$$

these equation can be solved by a standard iteration method in a suitable subspace of $\mathcal{C}_{1}$ ( $[8$ for details).

It is also possible to construct higher order iterated integrals starting from low order ones where for iterated integrals we means simply object which obey Chen's relations (44). Take for example 
the third order object $X^{a_{1} a_{2} a_{3}} \in \mathcal{C}_{2}$, eq. (4) can be written as a statement about the coboundary of $X^{a_{1} a_{2} a_{3}}$ :

$$
\delta X_{t u s}^{a_{1} a_{2} a_{3}}=X_{t u}^{a_{1} a_{2}} X_{u s}^{a_{3}}+X_{t u}^{a_{1}} X_{u s}^{a_{2} a_{3}}
$$

and in the above hypothesis on $X$ it is easy to check that the r.h.s. belongs to $\mathcal{Z C}_{2}^{3 \gamma} \subset \mathcal{Z C}_{2}^{1+}$ so that it is in the domain of $\Lambda$ and we can define

$$
X^{a_{1} a_{2} a_{3}}:=\Lambda\left[X^{a_{1} a_{2}} X^{a_{3}}+X^{a_{1}} X^{a_{2} a_{3}}\right]
$$

as the unique solution in $\mathcal{C}_{2}^{1+}$ of the Chen relation. Iteratively this allows to construct all the higher order iterated integrals. Some more work allows to prove a uniform estimate on the growth of the norms involved in the procedure [2, 8]:

$$
\left\|X^{\bar{a}}\right\|_{|\bar{a}| \gamma} \leq C_{1} \frac{C_{2}^{|\bar{a}|}}{(|\bar{a}| !)^{\gamma}}
$$

for any multiindex $a$.

\section{Trees}

What happens if $\gamma \leq 1 / 3$ ? The obstuction to the exactness of the increment $a$ in eq. (9) will no more belong to the domain of the sewing map. Indeed in eq. (7) we cannot anymore expect that the remainder belongs to $\mathcal{C}_{2}^{1+}$ and at the very least this would affect our argument for uniqueness. We are then forced to proceed and expand further the integral, or from another point of view, to add some counterterms to remove large contributions to $r$.

To understand the general structures that we need if we proceed further in the expansions it is better to take as working bench the more difficult case of the differential equation (2). The series solution will be indexed by rooted trees: a phenomenon which is present already for solutions to the ODE $d y / d t=f(y)[9,10]$.

\subsection{Labelled rooted trees}

Given a finite set $\mathcal{L}$, the $\mathcal{L}$-labeled rooted trees are the finite graphs where labels of $\mathcal{L}$ are attached to each vertex and where there is a special vertex called root such that there is a unique path from the root to any other vertex.

Some examples of rooted trees labeled by $\mathcal{L}=\{1,2,3\}$ are

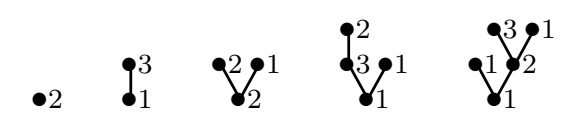

Trees does not distinguish the order of branches to each vertex. Given $k \mathcal{L}$-decorated rooted trees $\tau_{1}, \cdots, \tau_{k}$ and a label $a \in \mathcal{L}$ we define $\tau=\left[\tau_{1}, \cdots, \tau_{k}\right]_{a}$ as the tree obtained by attaching the $k$ roots of $\tau_{1}, \cdots, \tau_{k}$ to a new vertex with label $a$ which will be the root of $\tau$. Any decorated rooted tree can be constructed using the simple decorated tree $\bullet_{a}(a \in \mathcal{L})$ and the operation $[\cdots]$, e.g.

$$
[\bullet]=\text { ๖ } \quad[\bullet,[\bullet]]=\text { etc. }
$$


Denote $\mathcal{T}_{\mathcal{L}}$ the set of all $\mathcal{L}$ decorated rooted trees and let $\mathcal{T}$ the set of rooted trees without decoration (i.e. for which the set of labels $\mathcal{L}$ is made of a single element). There is a canonical map $\mathcal{T}_{\mathcal{L}} \rightarrow \mathcal{T}$ which simply forget all the labels and every function on $\mathcal{T}$ can be extended, using this map to a function on $\mathcal{T}_{\mathcal{L}}$ for any set of labels $\mathcal{L}$. Let $|\cdot|: \mathcal{T} \rightarrow \mathbb{R}$ the weight which counts the number of vertices of the (undecorated) tree defined recursively as

$$
|\bullet|=1, \quad\left|\left[\tau_{1}, \ldots, \tau_{k}\right]\right|=1+\left|\tau_{1}\right|+\cdots+\left|\tau_{k}\right|
$$

moreover the factorial $\gamma(\tau)$ of a tree $\tau$ is

$$
\gamma(\bullet)=1, \quad \gamma\left(\left[\tau_{1}, \ldots, \tau_{k}\right]\right)=\left|\left[\tau_{1}, \ldots, \tau_{k}\right]\right| \gamma\left(\tau_{1}\right) \cdots \gamma\left(\tau_{k}\right)
$$

finally the symmetry factor $\sigma$ is given by the recursive formula $\sigma(\tau)=1$ for $|\tau|=1$ and

$$
\sigma\left(\left[\tau^{1} \cdots \tau^{k}\right]_{a}\right)=\frac{k !}{\delta\left(\tau^{1}, \ldots, \tau^{k}\right)} \sigma\left(\tau^{1}\right) \cdots \sigma\left(\tau^{k}\right)
$$

where $\delta\left(\tau^{1}, \cdots, \tau^{k}\right)$ counts the number of different ordered $k$-uples $\left(\tau^{1}, \cdots, \tau^{k}\right)$ which corresponds to the same (unordered) collection $\left\{\tau^{1}, \cdots, \tau^{k}\right\}$ of subtrees. The factor $k ! / \delta\left(\tau^{1}, \ldots, \tau^{k}\right)$ counts the order of the subgroup of permutations of $k$ elements which does not change the ordered $k$-uple $\left(\tau^{1}, \cdots, \tau^{k}\right)$. Then $\sigma(\tau)$ is is the order of the subgroup of permutations on the vertex of the tree $\tau$ which do not change the tree (taking into account also the labels). Another equivalent recursive definition for $\sigma$ is

$$
\sigma\left(\left[\left(\tau^{1}\right)^{n_{1}} \cdots\left(\tau^{k}\right)^{n_{k}}\right]_{a}\right)=n_{1} ! \cdots n_{k} ! \sigma\left(\tau^{1}\right)^{n_{1}} \cdots \sigma\left(\tau_{k}\right)^{n_{k}}
$$

where $\tau^{1}, \ldots, \tau^{k}$ are distinct subtrees and $n_{1}, \ldots, n_{k}$ the respective multiplicities.

The algebra $\mathcal{A} \mathcal{T}_{\mathcal{L}}$ is the commutative free polynomial algebra generated by $\{1\} \cup \mathcal{T}_{\mathcal{L}}$ over $\mathbb{R}$, i.e. elements of $\mathcal{A} \mathcal{T}_{\mathcal{L}}$ are finite linear combination with coefficients in $\mathbb{R}$ of formal monomials in the form $\tau_{1} \tau_{2} \cdots \tau_{n}$ with $\tau_{1}, \ldots, \tau_{n} \in \mathcal{T}_{\mathcal{L}}$ or of the unit $1 \in \mathcal{A} \mathcal{T}_{\mathcal{L}}$. The tree monomials are called forests and are collectively denoted $\mathcal{F}_{\mathcal{L}}$; we include the empty forest $1 \in \mathcal{F}_{\mathcal{L}}$. The algebra $\mathcal{A} \mathcal{T}_{\mathcal{L}}$ is endowed with a graduation $g$ given by $g\left(\tau_{1} \cdots \tau_{n}\right)=\left|\tau_{1}\right|+\cdots+\left|\tau_{n}\right|$ and $g(1)=0$. This graduation induces a corresponding filtration of $\mathcal{A} \mathcal{T}_{\mathcal{L}}$ in finite dimensional linear subspaces $\mathcal{A}_{n} \mathcal{T}_{\mathcal{L}}$ generated by the set $\mathcal{F}_{\mathcal{L}}^{n}$ of forests of degree $\leq n$.

Any map $f: \mathcal{T}_{\mathcal{L}} \rightarrow A$ where $A$ is some commutative algebra, can be extended in a unique way to a homomorphism $f: \mathcal{A} \mathcal{T}_{\mathcal{L}} \rightarrow A$ by setting: $f\left(\tau_{1} \cdots \tau_{n}\right)=f\left(\tau_{1}\right) f\left(\tau_{2}\right) \cdots f\left(\tau_{n}\right)$.

In the following we will use letters $\tau, \rho, \sigma, \ldots$ to denote trees in $\mathcal{T}_{\mathcal{L}}$ or forests in $\mathcal{F}_{\mathcal{L}}$, the degree $g(\tau)$ of a forest $\tau \in \mathcal{F}_{\mathcal{L}}$ will also be written as $|\tau|$. Roman letters $a, b, c, \cdots \in \mathcal{L}$ will denote vector indexes (i.e. labels) while $\bar{a}, \bar{b}, \ldots$ will denote multi-indexes with values in $\mathcal{L}: \bar{a}=\left(a_{1}, \ldots, a_{n}\right) \in \mathcal{L}^{n}$ with $|\bar{a}|=n$ the size of this multi-index.

\subsection{Iterated integrals and the Connes-Kreimer Hopf algebra}

Given a smooth path $x \in C^{1}\left([0, T], \mathbb{R}^{n}\right)$ we can canonically associate to it a family of 2 -increments $X^{\tau}$ indexed by trees labelled by $\mathcal{L}=\{1, \ldots, n\}$ by the iterated integrals

$$
X_{t s}^{\bullet a}=x_{t}-x_{s}, \quad X_{t s}^{\left[\tau_{1} \cdots \tau_{n}\right]_{a}}=\int_{s}^{t} X_{u s}^{\tau_{1}} \cdots X_{u s}^{\tau_{n}} d x_{u}^{a}
$$

Iterated integrals like (3) corresponds to "linear" trees $\tau=\left[\left[\cdots\left[\bullet_{a_{n}}\right]_{a_{n-1}} \cdots\right]_{a_{2}}\right]_{a_{1}}$ and by abuse of notation we will continue to write $X^{a_{1} \cdots a_{n}}$ for such $X^{\tau}$. The generalization of the Chen's 
relations (4) involves the Hopf algebra structure on on labelled trees essentially introduced by Connes and Kreimer which we now brefly describe.

On the algebra $\mathcal{A} \mathcal{T}_{\mathcal{L}}$ we can define a counit $\varepsilon: \mathcal{A} \mathcal{T}_{\mathcal{L}} \rightarrow \mathbb{R}$ as an algebra homomorphism such that $\varepsilon(1)=1$ and $\varepsilon(\tau)=0$ otherwise and a coproduct $\Delta: \mathcal{A} \mathcal{T}_{\mathcal{L}} \rightarrow \mathcal{A} \mathcal{T}_{\mathcal{L}} \otimes \mathcal{A} \mathcal{T}_{\mathcal{L}}$ as the algebra homomorphism such that

$$
\Delta(\tau)=1 \otimes \tau+\sum_{a \in \mathcal{L}}\left(B_{+}^{a} \otimes \mathrm{id}\right)\left[\Delta\left(B_{-}^{a}(\tau)\right)\right]
$$

on trees $\tau \in \mathcal{T}_{\mathcal{L}}$, where $B_{+}^{a}(1)=\bullet_{a}$ and $B_{+}^{a}\left(\tau_{1} \cdots \tau_{n}\right)=\left[\tau_{1} \cdots \tau_{n}\right]_{a}$ and $B_{-}^{a}$ is the inverse of $B_{+}^{a}$ or is equal to zero if the tree root does not have label $a$, i.e.

$$
B_{-}^{a}\left(B_{+}^{b}\left(\tau_{1} \cdots \tau_{n}\right)\right)= \begin{cases}\tau_{1} \cdots \tau_{n} & \text { if } a=b \\ 0 & \text { otherwise. }\end{cases}
$$

Endowed with $\varepsilon$ and $\Delta$ the algebra $\mathcal{A} \mathcal{T}_{\mathcal{L}}$ become a bialgebra, there exists also an antipode $S$ which complete the definition of the Hopf algebra structure on $\mathcal{T}_{\mathcal{L}}$ as described by Connes-Kreimer [11] (in the unlabeled case).

We will often use Sweedler's notation for the coproduct $\Delta \tau=\sum \tau_{(1)} \otimes \tau_{(2)}$ but we also introduce a counting function $c: \mathcal{T}_{\mathcal{L}} \times \mathcal{T}_{\mathcal{L}} \times \mathcal{F}_{\mathcal{L}} \rightarrow \mathbb{N}$ such that $\Delta \tau=\sum_{\rho \in \mathcal{T}_{\mathcal{L}}, \sigma \in \mathcal{F}_{\mathcal{L}}} c(\tau, \rho, \sigma) \rho \otimes \sigma$ moreover it will be useful to consider also the reduced coproduct $\Delta^{\prime} \tau=\Delta \tau-1 \otimes \tau-\tau \otimes 1$ with counting function $c^{\prime}$.

The generalization of eq. (4) reads as follows:

Theorem 4.1 (Tree multiplicative property). The map $X$ satisfy the algebraic relation

$$
\delta X^{\sigma}=X^{\Delta^{\prime}(\sigma)}, \quad \sigma \in \mathcal{A} \mathcal{T}_{\mathcal{L}}
$$

Let us give an example in one dimension $(d=1)$ where trees are not decorated. The forests with $|\tau| \leq 3$ are

\section{$.8, . . \$, .8, \ldots .8 \%$}

The action of the reduced coproduct on these forests and the corresponding action of the coboundary on the iterated integrals are given by

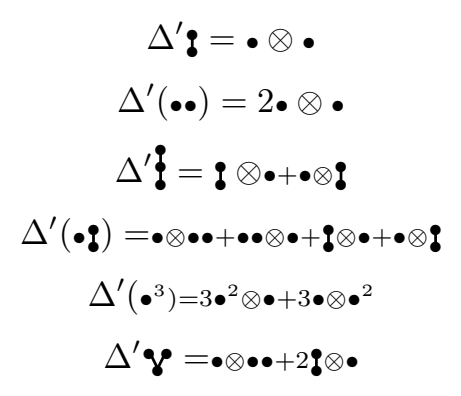

$$
\begin{aligned}
& \delta X_{\text {tus }}^{\boldsymbol{\ell}}=X_{t u}^{\bullet} X_{u s}^{\bullet} \\
& \delta X_{t u s}^{\bullet \bullet}=2 X_{t u}^{\bullet} X_{u s}^{\bullet}
\end{aligned}
$$

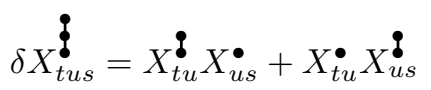

$$
\begin{aligned}
& \delta X_{t u s}^{\bullet \mathfrak{q}}=X_{t u}^{\bullet} X_{u s}^{\bullet \bullet}+X_{t u}^{\bullet \bullet} X_{u s}^{\bullet}+X_{t u}^{\mathfrak{\ell}} X_{u s}^{\bullet}+X_{t u}^{\bullet} X_{u s}^{\mathfrak{g}} \\
& \delta X_{\text {tus }}^{\bullet^{3}}=3 X_{\text {tu }}^{\bullet^{2}} X_{u s}^{\bullet}+3 X_{t u}^{\bullet} X_{u s}^{\bullet^{2}} \\
& \delta X_{t u s}^{\boldsymbol{\vartheta}}=X_{t u}^{\bullet} X_{u s}^{\bullet \bullet}+2 X_{t u}^{\boldsymbol{q}} X_{u s}^{\bullet}
\end{aligned}
$$

As a further example consider the iterated integrals $T^{\tau}$ associated to the identity path

$$
T_{t s}^{\bullet}=t-s, \quad T_{t s}^{\left[\tau_{1} \cdots \tau_{n}\right]}=\int_{s}^{t} T_{u s}^{\tau_{1}} \cdots T_{u s}^{\tau_{n}} d u
$$


By induction it is not difficult to prove that $T_{t s}^{\tau}=(t-s)^{|\tau|}(\tau !)^{-1}$, so applying Thm. 4.1 to $T^{\tau}$ we get a remarkable binomial-like formula for the Connes-Kreimer coproduct

$$
(a+b)^{|\tau|}=\sum \frac{\tau !}{\tau^{(1)} ! \tau^{(2) !}} a^{\left|\tau^{(1)}\right|} b^{\left|\tau^{(2)}\right|} .
$$

Iterated integrals of this sort appears naturally when trying to expand in series the solution of driven differential equations: for any analytic vectorfield $f: \mathbb{R}^{d} \rightarrow \mathbb{R}^{n}$ and any smooth path $x \in C^{1}\left([0, T], \mathbb{R}^{n}\right)$, the solution of the differential equation $d y_{t}=\sum_{a \in \mathcal{L}} f_{a}\left(y_{t}\right) d x_{t}^{a}$, with $y_{0}=\eta$ admit locally the series representation

$$
\delta y_{t s}=\sum_{\tau \in \mathcal{T}_{\mathcal{L}}} \frac{1}{\sigma(\tau)} \phi^{f}(\tau)\left(y_{s}\right) X_{t s}^{\tau}, \quad y_{0}=\eta
$$

where we recursively define functions $\phi^{f}(\tau)$ as

$$
\phi^{f}\left(\bullet_{a}\right)(\xi)=f_{a}(\xi), \quad \phi^{f}\left(\left[\tau^{1} \cdots \tau^{k}\right]_{a}\right)(\xi)=\sum_{\bar{b} \in \mathcal{I} \mathcal{L}_{1}:|\bar{b}|=k} f_{a ; b_{1} \ldots b_{k}}(\xi) \prod_{i=1}^{k}\left[\phi^{f}\left(\tau^{i}\right)(\xi)\right]^{b_{i}}
$$

Note that using the regularity of the path $x$ the iterated integrals $X^{\tau}$ can be simplified: indeed Chen [1] proved that products of iterated integrals can be always expressed as linear combination of iterated integrals via the shuffle product:

$$
X_{t s}^{a_{1} \cdots a_{n}} X_{t s}^{b_{1} \cdots b_{m}}=\sum_{\bar{c} \in \operatorname{Sh}(\bar{a}, \bar{b})} X_{t s}^{c_{1} \cdots c_{n+m}}
$$

where given two multi-indexes $\bar{a}=\left(a_{1}, \cdots, a_{n}\right)$ and $\bar{b}=\left(b_{1}, \cdots, b_{n}\right)$ their shuffles $\operatorname{Sh}(\bar{a}, \bar{b})$ is the set of all the possible permutations of the $(n+m)$-uple $\left(a_{1}, \ldots, a_{n}, b_{1}, \ldots, b_{m}\right)$ which does not change the ordering of the two subsets $\bar{a}, \bar{b}$. Using relation (18) every $X^{\tau}$ can be reduced to a linear combination of standard iterated integrals $\left\{X^{\bar{a}}\right\}_{\bar{a}}$.

It is however interesting that many costructions related to integrals and solution of driven differential equations do not depend on eq. (18) being valid. For example in the theory of the Itô integral eq. (18) does not hold and more complex algebraic relations have to be considered: if $x$ is a multidimensional Brownian motion and $X$ defined via Itô integration we have

$$
X_{t s}^{a} X_{t s}^{b}=X_{t s}^{a b}+X_{t s}^{b a}+\delta_{a b}(t-s) .
$$

Let us clarify the algebraic framework in which we consider possible integration theories. It turns out that the only data we really need to build a family $\left\{X^{\tau}\right\}_{\tau \in \mathcal{T}_{\mathcal{L}}}$ satisfying (15) is given by a set of linear maps $\left\{I^{a}\right\}_{a \in \mathcal{L}}$ defined on a domain $\mathcal{D}_{I} \subset \mathcal{C}_{2}^{+}$(the unital algebra obtained extending $\mathcal{C}_{2}$ with a unit $e$ and considering the point-wise product) to $\mathcal{C}_{2}$ satisfying two properties:

1. $I(h f)_{t s}=I(h)_{t s} f_{s}$ for all $h \in \mathcal{D}_{I}, f \in \mathcal{C}_{1}$ and where $(h f)_{t s}=h_{t s} f_{s}$; 
2. $\delta I(h)_{t u s}=I(e)_{t u} h_{u s}+\sum_{i} I\left(h^{1, i}\right)_{t u} h_{u s}^{2, i}$ when $h \in \mathcal{D}_{I}$ with $\delta h_{t u s}=\sum_{i} h_{t u}^{1, i} h_{u s}^{2, i}$ and $h^{1, i} \in \mathcal{D}_{I}$.

Then given a family $\left\{I^{a}\right\}_{a \in \mathcal{L}}$ of such integral maps on a common algebra $\mathcal{D} \subseteq \mathcal{C}_{2}$ we can associate to them a family $\left\{X^{\tau}\right\}_{\tau \in \mathcal{F}_{\mathcal{L}}}$ recursively as

$$
X_{t s}^{\bullet a}=I^{a}(e)_{t s}, \quad X_{t s}^{\left[\tau^{1} \cdots \tau^{k}\right]_{a}}=I^{a}\left(X^{\tau^{1} \cdots \tau^{k}}\right)_{t s}, \quad X_{t s}^{\tau^{1} \cdots \tau^{k}}=X_{t s}^{\tau^{1}} \cdots X_{t s}^{\tau^{k}} .
$$

In this way we estabilish an algebra homomorphism from $\mathcal{A} \mathcal{T}_{\mathcal{L}}$ to a subalgebra of $\mathcal{C}_{2}$ generated by the $X^{\tau}$-s. This homomorphism send the operation $B_{+}^{a}$ on $\mathcal{A} \mathcal{T}_{\mathcal{L}}$ to the integral map $I^{a}$ on $\mathcal{C}_{2}$ in such a way that Theorem 4.1 holds.

Given $X^{\tau}$ for any $\tau \in \mathcal{T}_{\mathcal{L}}$ with $|\tau| \leq n$ and such that $\left\|X^{\tau}\right\|_{\gamma|\tau|} \leq C$ for some constant $C$ and $\gamma \in(1 /(n+1), 1)$ we are able to extend to any $\tau$ with $|\tau|>n$ the "integrals" $X^{\tau} \in \mathcal{C}_{2}^{\gamma|\tau|}$ in a unique manner solving the equation $\delta X^{\tau}=X^{\Delta^{\prime} \tau}$ with the aid of the sewing map. This extension will satisfy the bound

$$
\left\|X^{\tau}\right\|_{\gamma|\tau|} \leq C_{1} C_{2}^{|\tau|} q_{\gamma}(\tau)
$$

for any $\tau$ with $C_{1}, C_{2}$ two finite constants and $q_{\gamma}(\tau)$ a function satisfying

$$
q_{\gamma}(\tau)=\frac{1}{2^{\gamma|\tau|}-2} \sum^{\prime} q_{\gamma}\left(\tau^{(1)}\right) q_{\gamma}\left(\tau^{(2)}\right)
$$

The actual asympotic behavior of $q_{\gamma}$ for $|\tau| \rightarrow \infty$ is not yet know but we conjecture that it should hold the equivalence

$$
q_{\gamma}(\tau) \simeq(\tau !)^{-\gamma}
$$

(see [5]). This asymptotic behavior is satisfied on the subset of linear trees and is consistent with our results on tree-indexed iterated integrals in the context of 3d Navier-Stokes equation [6] (see also Sect. 5).

We call the object $\left\{X^{\tau}\right\}_{\tau}$ satisfying (15) and (19) for some $\gamma \in(0,1)$ a $(\gamma$-) branched rough path. By the above consideration it is clear that it is determined by the finite subset $\left\{X^{\tau}\right\}_{\gamma|\tau| \leq 1}$.

Assuming that the integrals $\left\{I^{a}\right\}_{a \in \mathcal{L}}$ generate a $\gamma$-branched rough path in the above sense, then we can extend the integral to a larger class of integrands. Take a set of constants $\left\{h_{0}^{\tau}\right\}_{\tau}$ and consider the path $h \in \mathcal{C}_{1}$ defined as $h_{t}=\sum_{\tau} h_{0}^{\tau} X_{t 0}^{\tau}$, then

$$
\delta h_{t s}=\sum_{\tau} h_{0}^{\tau} \sum^{\prime} X_{t s}^{\tau^{(1)}} X_{s 0}^{\tau^{(2)}}=\sum_{\tau, \rho, \sigma} c^{\prime}(\tau, \rho, \sigma) h_{0}^{\tau} X_{t s}^{\rho} X_{s 0}^{\sigma}=\sum_{\tau} h_{s}^{\tau} X_{t s}^{\tau}
$$

where we introduced paths $h^{\tau} \in \mathcal{C}_{1}$ by $h_{s}^{\tau}=\sum_{\sigma, \rho, \tau} c^{\prime}(\rho, \tau, \sigma) h_{0}^{\rho} X_{s 0}^{\sigma}$. We must have

$$
0=\delta \sum_{\tau} X^{\tau} h^{\tau}=\sum_{\tau} X^{\tau^{(1)}} X^{\tau^{(2)}} h^{\tau}-X^{\tau} \delta h^{\tau}
$$

so we can check that

$$
\delta h^{\tau}=\sum_{\rho, \tau, \sigma} c^{\prime}(\rho, \tau, \sigma) X^{\sigma} h^{\rho}
$$

also holds. At this level these relations are formal since they require an infinite number of terms to hold, however exploiting our analysis of the $\left(\mathcal{C}_{*}, \delta\right)$ complex and the sewing map we can work modulo $\mathcal{C}_{2}^{1+}$ and neglect the terms in the expansions which involve $X^{\tau}$-s with $\gamma|\tau|>1$. A more 
accurate analysis (which can be found in [5]) reveals that for the purpose of integrating and solving differential equations we can actually work modulo larger terms but for the sake of clarity we refrain to do this here.

We call a controlled path (by $X$ ) any path $h$ which satisfy modulo $\mathcal{C}_{2}^{1+}$ the eq. (21) together with eq. (22) for all trees $\tau$ with $\gamma|\tau|<1$. The integral $I$ can then be extended to integrate any controlled path. Indeed since $h e=e h+\delta h$ it is a consistent definition to set

$$
\begin{aligned}
I^{a}(h) & =I^{a}(h e)=I^{a}(e) h+I^{a}(\delta h)=X^{\bullet} h+\sum_{\gamma|\tau|<1} I^{a}\left(X^{\tau}\right) h^{\tau}+I^{a}(\mathcal{R}) \\
& =X^{\bullet} h+\sum_{\gamma|\tau|<1} X^{[\tau]_{a}} h^{\tau}+\mathcal{R}
\end{aligned}
$$

where we exploited the properties of the integral $I^{a}$ and the definition of $X$ and where $\mathcal{R} \in \mathcal{C}_{2}^{1+}$ is a generic remainder term (possibly different from line to line). Controlled paths are also stable under mapping by sufficiently smooth functions $f$ (the degree of differentiability depends on $\gamma$ ): i.e. if $y$ is a controlled path, then $z=f(y)$ is again a controlled path with an explicit formula for the coefficients $z^{\tau}$ in term of the derivatives of $f$ and of the coefficients $y^{\tau}$. Using these properties we can consider the differential equation for the integrals $I$ and vectorfield $f$ :

$$
\delta y=I^{a}\left(f_{a}(y)\right), \quad y_{0}=\eta
$$

in the space of controlled paths $y$ and solve it by a fixed-point argument.

\section{Infinite dimensional dynamical systems}

In this section we would like to show how the ideas and the tools described before could be applied in the context of infinite dimensional dynamical system by introducing operator-valued iterated integrals (and rough paths) which by their non-commutative nature are intrinsically indexed by trees. In particular we will discuss two different examples: the 1d periodic Korteweg-de-Vries $(\mathrm{KdV})$ equation and the 3d Navier Stokes (NS) equation. In the first case we will exploit the increment complex to analyze perturbatively the solution for irregular initial data, in the second case we will use series expansion over trees of the solution to analyze the dynamics for large times .

As before we will restrict ourself to an overview of the results and to a sketch of the arguments. The interested readers can find a rigorous discussion elsewhere [7, 6] and he can refer to [12] for extension of these consideration covering the analysis of stochastic partial differential equations driven by irregular noises.

\subsection{The KdV equation}

The $1 \mathrm{~d}$ periodic $\mathrm{KdV}$ equation is the partial differential equation

$$
\partial_{t} u(t, \xi)+\partial_{\xi}^{3} u(t, \xi)+\frac{1}{2} \partial_{\xi} u(t, \xi)^{2}=0, \quad u(0, \xi)=u_{0}(\xi), \quad(t, \xi) \in \mathbb{R} \times \mathbb{T}
$$

on the torus $\mathbb{T}=[-\pi, \pi]$. When the initial data is not smooth this equation must be interpreted in the integral form

$$
u(t)=U(t) u_{0}+\frac{1}{2} \int_{0}^{t} U(t-s) \partial[u(s)]^{2} d s
$$


where $U$ is the "free propagator" given by the linear equation $\partial_{t} U(t)=\partial^{3} U(t), \partial$ denoting the spatial derivative, and where we consider the solution $u$ as a path in a space of functions. By going to the interaction picture $\tilde{v}_{t}=U(-t) u(t)$ we get

$$
\tilde{v}_{t}=u_{0}+\frac{1}{2} \int_{0}^{t} U(-s) \partial\left[U(s) \tilde{v}_{s}\right]^{2} d s
$$

Then the Fourier coefficients $\left\{v_{t}(k)\right\}_{k \in \mathcal{Z}}$ of $\tilde{v}_{t}$ satisfy the equation

$$
v_{t}(k)=v_{0}(k)+\frac{i k}{2} \sum_{k=k_{1}+k_{2}, k_{n} \neq 0} \int_{0}^{t} e^{-i\left(k^{3}-k_{1}^{3}-k_{2}^{3}\right) s} v_{s}\left(k_{1}\right) v_{s}\left(k_{2}\right) d s, \quad t \in[0, T], k \neq 0 .
$$

We restrict our attention to initial conditions such that $v_{0}(0)=0$. By calling $\dot{X}$ the bilinear operator in the r.h.s. this equation takes the abstract form

$$
v_{t}=v_{s}+\int_{s}^{t} \dot{X}_{\sigma}\left(v_{\sigma}, v_{\sigma}\right) d \sigma, \quad t, s \in[0, T] .
$$

where the paths take values in the Hilbert space $H_{\alpha}$ of complex Fourier coefficients $\varphi(k)$ with $\varphi(0)=0$ and $\varphi(-k)=\overline{\varphi(k)}$ endowed with the scalar product $\left\langle\varphi_{1}, \varphi_{2}\right\rangle_{\alpha}=\sum_{k \neq 0} k^{2 \alpha} \varphi_{1}(-k) \varphi_{2}(k)$.

By iteratively substituting the unknown in this integral equation we obtain an expansion whose first terms looks like

$$
\begin{aligned}
v_{t}=v_{s} & +\int_{s}^{t} d \sigma \dot{X}_{\sigma}\left(v_{s}, v_{s}\right)+2 \int_{s}^{t} d \sigma \dot{X}_{\sigma}\left(v_{s}, \int_{s}^{\sigma} d \sigma_{1} \dot{X}_{\sigma_{1}}\left(v_{s}, v_{s}\right)\right) \\
& +\int_{s}^{t} d \sigma \dot{X}_{\sigma}\left(\int_{s}^{\sigma} d \sigma_{1} \dot{X}_{\sigma_{1}}\left(v_{s}, v_{s}\right), \int_{s}^{\sigma} d \sigma_{2} \dot{X}_{\sigma_{2}}\left(v_{s}, v_{s}\right)\right) \\
& +4 \int_{s}^{t} d \sigma \dot{X}_{\sigma}\left(v_{s}, \int_{s}^{\sigma} d \sigma_{1} \dot{X}_{\sigma_{1}}\left(v_{s}, \int_{s}^{\sigma_{1}} d \sigma_{2} \dot{X}_{\sigma_{2}}\left(v_{s}, v_{s}\right)\right)+r_{t s}\right.
\end{aligned}
$$

where $r_{t s}$ stands for the remaining terms in the expansion. Denote with $\mathcal{B} \mathcal{T}$ the set of (unlabeled) planar rooted trees with at most two branches at each node. A planar tree is a rooted tree endowed with an ordering of the branches at each node. Then each of the terms in this expansion can be associated to a tree in $\mathcal{B} \mathcal{T}$ and we can define recursively multi-linear operators $X^{\tau}$ as

$$
\begin{gathered}
X_{t s}^{\bullet}\left(\varphi_{1}, \varphi_{2}\right)=\int_{s}^{t} \dot{X}_{\sigma}\left(\varphi_{1}, \varphi_{2}\right) d \sigma \\
X_{t s}^{\left[\tau^{1}\right]}\left(\varphi_{1}, \ldots, \varphi_{m+1}\right)=\int_{s}^{t} \dot{X}_{\sigma}\left(X_{\sigma s}^{\tau^{1}}\left(\varphi_{1}, \ldots, \varphi_{m}\right), \varphi_{m+1}\right) d \sigma
\end{gathered}
$$

and

$$
X_{t s}^{\left[\tau^{1} \tau^{2}\right]}\left(\varphi_{1}, \ldots, \varphi_{m+n}\right)=\int_{s}^{t} \dot{X}_{\sigma}\left(X_{\sigma s}^{\tau^{1}}\left(\varphi_{1}, \ldots, \varphi_{m}\right), X_{\sigma s}^{\tau^{2}}\left(\varphi_{m+1}, \ldots, \varphi_{m+n}\right)\right) d \sigma
$$

Eq. (25) has the form

$$
\delta v_{t s}=X^{\bullet}\left(v^{\times 2}\right)_{t s}+X^{\boldsymbol{\xi}}\left(v^{\times 3}\right)_{t s}+X^{\boldsymbol{\xi}}\left(v^{\times 4}\right)_{t s}+X^{\boldsymbol{\vartheta}}\left(v^{\times 4}\right)_{t s}+r_{t s}
$$


as an equation in $\mathcal{C}_{2}$ where increments take values in $H_{\alpha}$ and where we let $v_{s}^{\times n}=\left(v_{s}, \ldots, v_{s}\right)(n$ times). The operators $X^{\tau}$ satisfy multiplicative relations

$$
\begin{aligned}
& \delta X^{\boldsymbol{\ell}}\left(\varphi_{1}, \varphi_{2}, \varphi_{3}\right)=X^{\bullet}\left(X^{\bullet}\left(\varphi_{1}, \varphi_{2}\right), \varphi_{3}\right),
\end{aligned}
$$

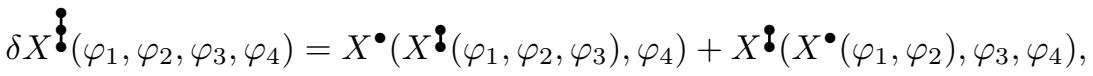

and

$$
\begin{aligned}
\delta X \boldsymbol{\bullet}\left(\varphi_{1}, \varphi_{2}, \varphi_{3}, \varphi_{4}\right)=X^{\bullet}\left(X^{\bullet}\left(\varphi_{1}, \varphi_{2}\right), X^{\bullet}\left(\varphi_{3}, \varphi_{4}\right)\right) \\
+X^{\boldsymbol{\natural}}\left(\varphi_{1}, \varphi_{2}, X^{\bullet}\left(\varphi_{3}, \varphi_{4}\right)\right)+X^{\boldsymbol{\natural}}\left(\varphi_{3}, \varphi_{4}, X^{\bullet}\left(\varphi_{1}, \varphi_{2}\right)\right)
\end{aligned}
$$

where we used the symmetry of the operator $\dot{X}$ to obtain this last equation. These relations have much in common with the analogous relations for branched rough paths, however here the additional information of the position of the various arguments must be taken into account in the combinatorics of the reduced coproduct. It would be interesting to determine a Hopf algebra structure on $\mathcal{B} \mathcal{T}$ which could account this additional information in a general way.

Note that $\dot{X}$ is unbounded on $H_{\alpha}$ while it is possible to prove that $X^{\bullet}$ and $X^{\boldsymbol{\ell}}$ are bounded, in particular for the simplest of them, $X^{\bullet}$, we have the bound

$$
\left|X_{t s}^{\bullet}\left(\varphi_{1}, \varphi_{2}\right)\right|_{\alpha} \leq C|t-s|^{\gamma}\left|\varphi_{1}\right|_{\alpha}\left|\varphi_{2}\right|_{\alpha}
$$

with the parameters $\gamma$ and $\alpha$ satisfying

$$
\gamma<1 / 2, \alpha \in \mathbb{R} \text { with } \gamma-\alpha<1 \text { and } 3 \gamma-\alpha<3 / 2 \text {. }
$$

Moreover we are able to decompose the second member of the hierarchy $X^{\boldsymbol{t}}$ as

$$
X^{\boldsymbol{\ell}}\left(\varphi_{1}, \varphi_{2}, \varphi_{3}\right)=\widehat{X}^{\boldsymbol{\ell}}\left(\varphi_{1}, \varphi_{2}, \varphi_{3}\right)+\Phi\left(\varphi_{1}, \varphi_{2}\right) \varphi_{3}+\Phi\left(\varphi_{1}, \varphi_{3}\right) \varphi_{2}
$$

where for the same range of $\gamma, \alpha$ as in (27) we have

$$
\left|\hat{X}_{t s}^{\boldsymbol{d}}\left(\varphi_{1}, \varphi_{2}, \varphi_{3}\right)\right|_{\alpha} \leq C|t-s|^{2 \gamma}\left|\varphi_{1}\right|_{\alpha}\left|\varphi_{2}\right|_{\alpha}\left|\varphi_{3}\right|_{\alpha}
$$

while the operator $\Phi_{t s}: H_{\alpha} \times H_{\alpha} \rightarrow \mathcal{C}$ is bounded only for $\alpha \geq-1 / 2$ and

$$
\left|\Phi_{t s}\left(\varphi_{1}, \varphi_{2}\right)\right| \leq C|t-s|\left|\varphi_{1}\right|_{\alpha}\left|\varphi_{2}\right|_{\alpha} .
$$

The analysis of the higher order operators has not yet been performed. However already at this stage something can be said if we take $3 \gamma>1$ since we are naturally led to consider eq. (26) as a increment equation and rewrite it using the sewing map and the operators $X$ (only up to second order) obtaining the equation

$$
\delta v=(1-\Lambda \delta)\left[X^{\bullet}\left(v^{\times 2}\right)+X^{\boldsymbol{\imath}}\left(v^{\times 3}\right)\right]
$$

which can be solved by fixed point methods in $H_{\alpha}$ for any $\alpha>-1 / 2$ (cfr. (27) and the condition on $\Phi)$.

The condition $\alpha \geq-1 / 2$ is essentially imposed by the operator $\Phi$ appearing in the decomposition (28) of $X^{\boldsymbol{d}}$. This constraint it is linked with a resonance phenomenon which apperars in the 
scattering by the non-linear term involving four waves and which hint to the fact that the KdV equation is not uniformly wellposed in $H_{\alpha}$ for $\alpha<-1 / 2$ [13, 14.

We have replaced the differential and integral approach to the study of this equation by an approach based on an operator valued rough path and the increment complex. It is interesting then to look how the properties of the dynamical system reflect in this unusual approach. As an example let us consider conservation laws.

The KdV equation formally conserves the $H_{0}$ norm. This conservation law imposes additional algebraic relations to the operators $X$ : it is not difficult to prove that we have

$$
\left\langle\varphi_{1}, X_{t s}^{\bullet}\left(\varphi_{2}, \varphi_{3}\right)\right\rangle_{0}+\left\langle\varphi_{2}, X_{t s}^{\bullet}\left(\varphi_{1}, \varphi_{3}\right)\right\rangle_{0}+\left\langle\varphi_{3}, X_{t s}^{\bullet}\left(\varphi_{2}, \varphi_{1}\right)\right\rangle_{0}=0
$$

and that

$$
2\left\langle\varphi, X_{t s}^{2}(\varphi, \varphi, \varphi)\right\rangle_{0}+\left\langle X_{t s}(\varphi, \varphi), X_{t s}(\varphi, \varphi)\right\rangle_{0}=0
$$

where all the test functions belong to $H_{0}$. To see that these two relations imply the $H_{0}$ conservation law for solutions we will prove that $\delta\langle v, v\rangle_{0}=0$ when $v$ satisfy

$$
\delta v=X^{\bullet}\left(v^{\times 2}\right)+X^{\bullet}\left(v^{\times 3}\right)+\mathcal{R}
$$

cfr. eq. (29). Let us compute explicitly $\left[\delta\langle v, v\rangle_{0}\right]_{t s}=\left\langle v_{t}, v_{t}\right\rangle_{0}-\left\langle v_{s}, v_{s}\right\rangle_{0}=2\left\langle\delta v_{t s}, v_{s}\right\rangle_{0}+\left\langle\delta v_{t s}, \delta v_{t s}\right\rangle_{0}$ . Substituting in this expression the equation (32) we get

$$
\begin{aligned}
{\left[\delta\langle v, v\rangle_{0}\right]_{t s}=2\langle} & \left.X_{t s}^{\bullet}\left(v_{s}, v_{s}\right)+X_{t s}^{\mathfrak{d}}\left(v_{s}, v_{s}, v_{s}\right), v_{s}\right\rangle_{0} \\
& +\left\langle X_{t s}^{\bullet}\left(v_{s}, v_{s}\right), X_{t s}^{\bullet}\left(v_{s}, v_{s}\right)\right\rangle_{0}+\mathcal{R} .
\end{aligned}
$$

The relation (30) implies that $\left\langle v_{s}, X_{t s}^{\bullet}\left(v_{s}, v_{s}\right)\right\rangle_{0}=0$ while eq. (31) allows to cancel the $X^{\boldsymbol{\ell}}$ term with the quadratic $X^{\bullet}$ term. After the cancellations the increment of the $H_{0}$ norm squared is then $\left[\delta\langle v, v\rangle_{0}\right] \in \mathcal{C}_{2}^{1+}$ but this means that it must be zero and that $\left\langle v_{t}, v_{t}\right\rangle_{0}=\left\langle v_{0}, v_{0}\right\rangle_{0}$ for any $t \geq 0$.

\subsection{Navier-Stokes-like equations}

We consider the NS equation in $\mathbb{R}^{3}$ which going in Fourier space can be written

$$
v_{t}(k)=e^{-|k|^{2} t} v_{0}(k)+i \int_{0}^{t} e^{-|k|^{2}(t-s)} \int_{\mathbb{R}^{3}} d k^{\prime}\left\langle k, v_{s}\left(k-k^{\prime}\right)\right\rangle P_{k} v_{s}\left(k^{\prime}\right) d s
$$

where $v_{t}$ is the Fourier transform of the velocity field, $\langle\cdot, \cdot\rangle$ is the scalar product in $\mathbb{C}^{3}$ and $P_{k}: \mathcal{C}^{3} \rightarrow$ $\mathcal{C}^{3}$ is the projection on the directions orthogonal to the vector $k \in \mathbb{R}^{3}$, i.e. $P_{k} a=a-\langle k, a\rangle k|k|^{-1}$. Eq. (33) will be studied in the spaces $\Phi(\alpha), \alpha \in[2,3)$ where $v \in \Phi(\alpha)$ if $v \in C\left(\mathbb{R}^{3} ; \mathcal{C}^{3}\right)$ with $k \cdot v(k)=0$ and $\|v\|_{\alpha}=\sup _{k \in \mathbb{R}^{3}}|k|^{\alpha}|v(k)|<\infty$. We will write $\alpha=2+\varepsilon$ with $\varepsilon \in[0,1)$. The spaces $\Phi(\alpha)$ can contain solutions with infinite energy and enstrophy so classical results about existence and uniqueness do not apply. Sinai [15, 16, 17, studied eq. (33) in $\Phi(\alpha)$ with $\alpha>2$, showing that there is existence of unique local solutions and that these solutions survive for arbitrary large time if the initial condition is small enough. Related works on NS are those of Le Jan and Sznitman 18, Cannone and Planchon [19] and the reviews of Bhattacharya et al. in [20] and Waymire [21].

Following [6] we will describe the representation for these solutions as series indexed by planar binary trees. The use of trees to rigoroulsy analyze the NS equation has been somewhat pioneered by Gallavotti [22]. 
The NS equation can be cast in the abstract form

$$
u_{t}=S_{t} u_{0}+\int_{0}^{t} S_{t-s} B\left(u_{s}, u_{s}\right) d s
$$

where $S$ is a bounded semi-group on $\Phi(\alpha)$ and $B$ is a symmetric bilinear operator which is usually defined only on a subspace of $\Phi(\alpha)$. Here we cannot proceed as in the KdV case by going to the interaction picture since $S$ is only a semi-group, so we must cope with the convolution directly. In [6] we showed that the solutions of this equation in the case of the $3 \mathrm{~d}$ NS equation have the norm convergent series representation

$$
u_{t}=S_{t} u_{0}+\sum_{\tau \in \mathcal{B} \mathcal{T}} X_{t 0}^{\tau}\left(u_{0}^{\times \theta(\tau)}\right)
$$

where $\theta(\tau)$ is a degree function defined by $\theta(\bullet)=2, \theta([\tau])=1+\theta(\tau), \theta\left(\left[\tau_{1} \tau_{2}\right]\right)=\theta\left(\tau^{1}\right)+\theta\left(\tau^{2}\right)$ and the $\theta(\tau)$-multilinear operators $X^{\tau}$ have recursive definition

$$
\begin{gathered}
X_{t s}^{\bullet}\left(\varphi^{\times 2}\right)=\int_{s}^{t} S_{t-u} B\left(S_{u-s} \varphi, S_{u-s} \varphi\right) d u \\
X_{t s}^{\left[\tau^{1}\right]}\left(\varphi^{\times\left(\theta\left(\tau^{1}\right)+1\right)}\right)=\int_{s}^{t} S_{t-u} B\left(X_{u s}^{\tau^{1}}\left(\varphi^{\times \theta\left(\tau^{1}\right)}\right), \varphi\right) d u
\end{gathered}
$$

and

$$
X_{t s}^{\left[\tau^{1} \tau^{2}\right]}\left(\varphi^{\times\left(\theta\left(\tau^{1}\right)+\theta\left(\tau^{2}\right)\right)}\right)=\int_{s}^{t} S_{t-u} B\left(X_{u s}^{\tau^{1}}\left(\varphi^{\times \theta\left(\tau^{1}\right)}\right), X_{u s}^{\tau^{2}}\left(\varphi^{\times \theta\left(\tau^{2}\right)}\right)\right) d u
$$

and by induction we can prove that, for any $\varepsilon \in[0,1)$ these operators are bounded by

$$
\left|X_{t s}^{\tau}\left(h^{\theta(\tau)}\right)(k)\right| \leq C_{\tau} \frac{e^{-|k|^{2}(t-s) /(|\tau|+1)}}{|k|^{\alpha}}(t-s)^{|\tau| \varepsilon / 2}\|h\|_{\alpha}^{\theta(\tau)}
$$

where the constants $C_{\tau}$ can be chosen as

$$
C_{\tau}=A^{|\tau|}(\tau !)^{-\varepsilon / 2}
$$

for some other constant $A>0$ depending only on $\varepsilon$.

Due to the presence of the convolution integral these $X$ operators does not behaves nicely with respect to the coboundary $\delta$. In [12] we introduced cochain complex $\left(\hat{C}_{*}, \tilde{\delta}\right)$ adapted to the study of such convolution integrals where the coboundary $\tilde{\delta}$ is obtained from $\delta$ by a "twisting" involving the semigroup. There exists also a corresponding convolutional sewing map $\tilde{\Lambda}$ which provide an appropriate inverse to $\tilde{\delta}$. Algebraic relations for these iterated integrals have then by-now familiar expressions, e.g.:

$$
\tilde{\delta} X^{\mathfrak{\ell}}\left(\varphi^{\times 3}\right)=X^{\bullet}\left(X^{\bullet}\left(\varphi^{\times 2}\right), S \varphi\right)
$$

and so on. Indeed $X$ can be considered as a branched rough path and the asymptotic behaviour (37) supports somewhat the conjectured asymptotics (20).

The series representation (35) together with the bounds (36) imply that

$$
\left|u_{t}(k)\right| \leq\left|S_{t} u_{0}(k)\right|+\sum_{\tau \in \mathcal{B} \mathcal{T}} \frac{B^{|\tau|}}{\sigma(\tau) \tau !} \frac{e^{-\left(k^{2} t\right) /(|\tau|+1)}}{|k|^{\alpha}} t^{\varepsilon|\tau| / 2}\left\|u_{0}\right\|_{\alpha}^{\theta(\tau)}
$$


By induction we can prove that $\gamma(\tau) \geq 2^{|\tau|-1}$ where equality holds for the binary trees for which every path from the root to the leaves has the same length. This estimate together with

$$
(|\tau|+1) / 2 \leq \theta(\tau) \leq|\tau|+1
$$

easily proven by induction on $|\tau|$ give

$$
\left|u_{t}(k)\right| \leq\left|S_{t} u_{0}(k)\right|+\sum_{n \geq 1} Z_{n} B^{n} \frac{e^{-\left(k^{2} t\right) /(n+1)}}{|k|^{\alpha}} t^{\varepsilon n / 2}\left\|u_{0}\right\|_{\alpha}^{(n+1) / 2}\left(1+\left\|u_{0}\right\|_{\alpha}\right)^{(n+1) / 2}
$$

for some different constant $B$ and where $Z_{n}$ is the number of trees in $\mathcal{B T}$ with $n$ vertices for which we have the estimate $Z_{n} \leq D^{n}(n+1)^{-3 / 2}$ for a constant $D>0$. So the series (35) is controlled by the geometric series (40) and converges in norm $t$ is small or $\|h\|_{\alpha}$ is small. In the case $\varepsilon=0$ the dependence in time of the r.h.s. is bounded and so the series converges for all time if the initial condition is small enough. The series gives also additional informations on the global solution when $\varepsilon=0$ :

a) dissipation: for fixed $k \in \mathbb{R}^{3} \backslash\{0\}, \lim _{t \rightarrow \infty}\left|u_{t}(k)\right|=0$;

b) smoothness: for fixed $t>0$, there exists two constants $C_{3}, C_{4}$ such that $\left|v_{t}(k)\right| \leq C_{3} e^{-C_{4}|k| \sqrt{t}}$ as $|k| \rightarrow \infty$.

In particular this second property can be proved by the Laplace method applied to the majorizing series 40 .

Another interesting way to analyze the NS series (35) is to note that different classes of trees give different contributions. We define simple trees the trees with at most one branch at each vertex, i.e. of the form $[\cdots[\bullet] \cdots]$. Short trees are instead trees for which at each vertex we have two branches, each of which carries (asymptotically) a fixed proportion $(\alpha$ or $1-\alpha)$ of the vertice and without loosing generality we consider $\alpha \in(0,1 / 2)$. We will denote $\mathcal{B T}_{0}$ the set of simple trees and $\mathcal{B} \mathcal{T}_{\alpha}$ the set of short trees corresponding to the proportion $\alpha$. The distinction between these classes of trees is relevant when discussing the asymptotic behavior of the tree factorial. Indeed for $\tau \in \mathcal{B T}_{0}$ we have $\tau !=|\tau|$ ! while for any $\alpha \in(0,1 / 2)$ there exists constants $D_{1}, D_{2}, D_{3}, D_{4}$ such that, for any $\tau \in \mathcal{B} \mathcal{T}_{\alpha}$ we have

$$
D_{3}|\tau|^{-1} D_{4}^{|\tau|} \leq \gamma(\tau) \leq D_{1}|\tau|^{-1} D_{2}^{|\tau|}
$$

This different behavior is responsible for different convergence properties of the sum (35) when restricted to simple or short trees. Indeed it is possible to prove that the series restricted to simple trees is convergent for all times whatever the size of the initial condition while the estimate for short trees do not ensure this important property. In some sense the difficulty of finding global solution of NS is due to the presence of arbitrarily large short trees in the expansion. A similar phenomenon is observed in [17] and exploited in [23] to prove blow-up for the complex Navier-Stokes equation by a renormalization group argument.

\section{References}

[1] Kuo Tsai Chen. Iterated path integrals. Bull. Amer. Math. Soc., 83(5):831-879, 1977. 
[2] Terry J. Lyons. Differential equations driven by rough signals. Rev. Mat. Iberoamericana, 14(2):215-310, 1998.

[3] Terry Lyons and Zhongmin Qian. System control and rough paths. Oxford Mathematical Monographs. Oxford University Press, Oxford, 2002. Oxford Science Publications.

[4] Antoine Lejay. An introduction to rough paths. In Séminaire de Probabilités XXXVII, volume 1832 of Lecture Notes in Math., pages 1-59. Springer, Berlin, 2003.

[5] M. Gubinelli. Ramification of rough paths. J. Diff. Eq., 2008. to appear.

[6] Massimiliano Gubinelli. Rooted trees for 3D Navier-Stokes equation. Dyn. Partial Differ. Equ., $3(2): 161-172,2006$.

[7] M. Gubinelli. Rough solutions of the periodic Korteweg-de Vries equation. 2006.

[8] M. Gubinelli. Controlling rough paths. J. Funct. Anal., 216(1):86-140, 2004.

[9] J. C. Butcher. An algebraic theory of integration methods. Math. Comp., 26:79-106, 1972.

[10] Ch. Brouder. Trees, renormalization and differential equations. BIT, 44(3):425-438, 2004.

[11] Alain Connes and Dirk Kreimer. Hopf algebras, renormalization and noncommutative geometry. Comm. Math. Phys., 199(1):203-242, 1998.

[12] M. Gubinelli and S. Tindel. Rough evolution equations. 2006.

[13] Michael Christ, James Colliander, and Terrence Tao. Asymptotics, frequency modulation, and low regularity ill-posedness for canonical defocusing equations. Amer. J. Math., 125(6):1235$1293,2003$.

[14] J. Colliander, M. Keel, G. Staffilani, H. Takaoka, and T. Tao. Sharp global well-posedness for $\mathrm{KdV}$ and modified KdV on $\mathbb{R}$ and T. J. Amer. Math. Soc., 16(3):705-749 (electronic), 2003.

[15] Ya. G. Sinai. On local and global existence and uniqueness of solutions of the 3D Navier-Stokes system on $\mathbb{R}^{3}$. In Perspectives in analysis, volume 27 of Math. Phys. Stud., pages 269-281. Springer, Berlin, 2005.

[16] Yakov Sinai. Power series for solutions of the $3 D$-Navier-Stokes system on $\mathbf{R}^{3}$. J. Stat. Phys., 121(5-6):779-803, 2005.

[17] Ya. G. Sinal. A diagrammatic approach to the 3D Navier-Stokes system. Uspekhi Mat. Nauk, $60(5(365)): 47-70,2005$.

[18] Y. Le Jan and A. S. Sznitman. Stochastic cascades and 3-dimensional Navier-Stokes equations. Probab. Theory Related Fields, 109(3):343-366, 1997.

[19] Marco Cannone and Fabrice Planchon. On the regularity of the bilinear term for solutions to the incompressible Navier-Stokes equations. Rev. Mat. Iberoamericana, 16(1):1-16, 2000.

[20] Rabi N. Bhattacharya, Larry Chen, Scott Dobson, Ronald B. Guenther, Chris Orum, Mina Ossiander, Enrique Thomann, and Edward C. Waymire. Majorizing kernels and stochastic cascades with applications to incompressible Navier-Stokes equations. Trans. Amer. Math. Soc., 355(12):5003-5040 (electronic), 2003. 
[21] Edward C. Waymire. Probability \& incompressible Navier-Stokes equations: an overview of some recent developments. Probab. Surv., 2:1-32 (electronic), 2005.

[22] Giovanni Gallavotti. Foundations of fluid dynamics. Texts and Monographs in Physics. Springer-Verlag, Berlin, 2002. Translated from the Italian.

[23] Dong Li and Ya. G. Sinai. Blow ups of complex solutions of the 3D Navier-Stokes system and renormalization group method. J. Eur. Math. Soc. (JEMS), 10(2):267-313, 2008. 